Forschende

Komplementärmedizin und Klassische Naturheilkunde

\section{Tagungen und Kongresse - Meetings and Conferences}

Forsch Komplementärmed Klass Naturheilkd 2005;12:306-307

06.10.-08.10.2005
Berlin
Deutschland

$\ldots \ldots \ldots \ldots \ldots \ldots \ldots$

\subsection{0.-22.10.2005}

Hamburg

Deutschland

\section{-.................. \\ 27.10.2005}

Baden

Schweiz

\subsubsection{5}

Lausanne

Schweiz
Phytopharmaka und Phytotherapie - Forschung und Praxis

3. Gemeinsamer Kongress der Deutschen Gesellschaft für Klinische

Pharmakologie und Therapie, der Gesellschaft für Phytotherapie und der Gesellschaft für Arzneipflanzenforschung

3. Gesundheitspflege-Kongress

Kurs 5: Phytotherapie bei Erkrankungen der Atemwege

Phytothérapie et appareil locomoteur, traitement de la douleur

\section{Medizinische Woche}

Baden-Baden

Deutschland

\section{...................}

03.11.2005

Zürich

Schweiz

\subsection{1.-18.11.2005}

Szeged

Ungarn

\subsubsection{5 \\ Lausanne}

Schweiz

\subsubsection{5}

Wädenswil

Schweiz

\subsubsection{5 \\ Jena}

Deutschland

\section{Symposium zur Ratio und Plausibilität in der Naturheilkunde} «Der Darm - Dirigator über leibliches Wohl und Übel»
1. International Balnelogical and Medical Hydrological Course ISMH (International Society of Medical Hydrology)

1ère Journée Romande de Phytothérapie Médicale
"Arzt und Patient - Verantwortlichkeit und Partnerschaft"

Auskunft: Prof. Dr. med. Volker Schulz Oranienburger Chaussee 25, D-13465 Berlin

Tel. +49 30 40-12689, Fax -637973

E-mail v.schulz.berlin@t-online.de

Auskunft: Congress Partner $\mathrm{GmbH}$

Markgrafenstr. 56, D-10117 Berlin-Mitte

Tel. +49 30 2045-90, Fax -950

E-mail berlin@cpb.de

Auskunft: SMGP, Kurs-Sekretariat, Irène Tinguely

Hochschule Wädenswil, Grünental

Postfach 335, CH-8820 Wädenswil

Tel. +41 44 78999-80, Fax -50

E-mail kurse-smgp@hsw.ch

www.smgp.ch

Auskunft: SMGP, Kurs-Sekretariat, Irène Tinguely

Hochschule Wädenswil, Grünental

Postfach 335, CH-8820 Wädenswil

Tel. +41 44 78999-80, Fax -50

E-mail kurse-smgp@hsw.ch

www.smgp.ch

Auskunft: Pressestelle EHK, Beate Schweizer

Postfach 301120, D-70451 Stuttgart

Tel. +49 711 8931-295, Fax -167

E-mail Beate.Schweizer@thieme.de

Auskunft: UniversitätsSpital Zürich

Dept. für Innere Medizin, Institut für Naturheilkunde

Rämistr. 100, CH-8091 Zürich

Tel. +44 255-2460, Fax -4394

E-mail angela.stoerl@usz.ch

Auskunft: C\&T Hungary Ltd.

Congress Bureau \& Travel Agency

P.O.Box 898, H-6701 Szeged

Tel./Fax +36 62548485

E-mail congress@congresstravel.hu

www.congresstravel.hu

Auskunft: SMGP, Kurs-Sekretariat, Irène Tinguely

Hochschule Wädenswil, Grünental

Postfach 335, CH-8820 Wädenswil

Tel. +41 44 78999-80, Fax -50

E-mail kurse-smgp@hsw.ch

www.smgp.ch

Auskunft: SMGP, Kurs-Sekretariat, Irène Tinguely

Hochschule Wädenswil, Grünental

Postfach 335, CH-8820 Wädenswil

Tel. +41 44 78999-80, Fax -50

E-mail kurse-smgp@hsw.ch

www.smgp.ch

Auskunft: Prof. Dr. C. Uhlemann

Kompetenzzentrum Naturheilverfahren der Klinik für Innere Medizin II

Friedrich-Schiller-Universität Jena

Bachstr. 18, D-07740 Jena

Tel. +49 3641 933-180, Fax -182

E-mail Christine.Uhlemann@med.uni-jena.de 
30.03.2006

Solothurn

Schweiz

\subsection{5 .2006}

Wädenswil

Schweiz

\section{...............}

12.05.-14.05.200

Friedrichshafen

Deutschland

\subsection{6.-10.06.2006}

Engelberg

Schweiz

\subsubsection{6}

Wädenswil

Schweiz

\subsubsection{6}

Wädenswil

Schweiz

\subsubsection{6}

Baden

Schweiz

[...............

26.01.2007

Wädenswil

Schweiz

\section{COSMOPHARMA}

\section{Kurs 1: Grundkurs \\ Kurs 1: Grundkurs}

Kurs 8: Erkrankungen des Bewegungsapparates und Schmerztherapie

\section{Kurs 9: Phytotherapie in der Dermatologie}

Jahrestagung: Phytotherapie in der Palliativ- und Supportivmedizin

Kurs 10: Der individuell abgestimmte Gesamtbehandlungsplan
Auskunft: SMGP, Kurs-Sekretariat, Irène Tinguely

Hochschule Wädenswil, Grünental

Postfach 335, CH-8820 Wädenswil

Tel. +41 44 78999-80, Fax -50

E-mail kurse-smgp@hsw.ch

www.smgp.ch

Auskunft: SMGP, Kurs-Sekretariat, Irène Tinguely

Hochschule Wädenswil, Grünental

Postfach 335, CH-8820 Wädenswil

Tel. +41 44 78999-80, Fax -50

E-mail kurse-smgp@hsw.ch

www.smgp.ch

Auskunft: ZÄN Geschäftsstelle

Frau Gabi Möhrle

Am Promenadenplatz 1, D-72250 Freudenstadt

Tel. +49 7441 91858-0, Fax -22

www.zaen.org

Auskunft: SMGP,

Hochschule Wädenswil, Grünenta

Postfach 335, CH-8820 Wädenswil

Tel. +41 44 78999-80, Fax -50

E-mail kurse-smgp@hsw.ch

www.smgp.ch

Auskunft: SMGP, Kurs-Sekretariat, Irène Tinguely

Hochschule Wädenswil, Grünental

Postfach 335, CH-8820 Wädenswil

Tel. +41 44 78999-80, Fax -50

E-mail kurse-smgp@hsw.ch

www.smgp.ch

Auskunft: SMGP, Kurs-Sekretariat, Irène Tinguely

Hochschule Wädenswil, Grünental

Postfach 335, CH-8820 Wädenswil

Tel. +41 44 78999-80, Fax -50

E-mail kurse-smgp@hsw.ch

www.smgp.ch

Auskunft: SMGP, Kurs-Sekretariat, Irène Tinguely

Hochschule Wädenswil, Grünental

Postfach 335, CH-8820 Wädenswil

Tel. +41 44 78999-80, Fax -50

E-mail kurse-smgp@hsw.ch

www.smgp.ch

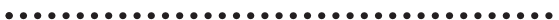

Auskunft: SMGP, Kurs-Sekretariat, Irène Tinguely

Hochschule Wädenswil, Grünental

Postfach 335, CH-8820 Wädenswil

Tel. +41 44 78999-80, Fax -50

E-mail kurse-smgp@hsw.ch

www.smgp.ch 